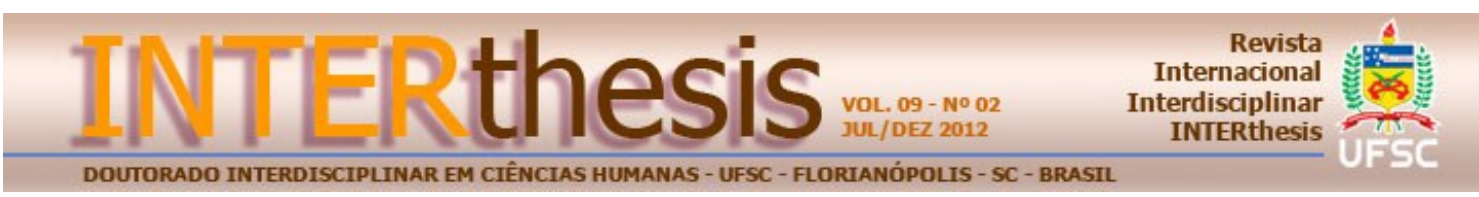

\title{
ANÁLISE DA TEMPERATURA DO AR E DA UMIDADE RELATIVA: ESTUDO DE MICROCLIMAS
}

\section{ANALYSIS OF AIR TEMPERATURE AND RELATIVE HUMIDITY: STUDY OF MICROCLIMATES}

\section{ANÁLISIS DE LA TEMPERATURA AMBIENTE $Y$ HUMEDAD RELATIVA: ESTUDIO DE MICROCLIMAS}

\author{
Elis Dener Lima Alves ${ }^{1}$ \\ Marcelo Sacardi Biudes ${ }^{2}$
}

\section{Resumo:}

A compreensão da variabilidade dos elementos do clima no tempo e no espaço é fundamental para o entendimento da dinâmica microclimática. Dessa forma, o objetivo deste trabalho foi analisar a variabilidade da temperatura do ar e da umidade relativa no campus de Cuiabá da Universidade Federal de Mato Grosso, e, por meio da técnica de agrupamento, analisar a formação dos grupos para propor um zoneamento microclimático na área de estudo. Para tanto, realizou-se no ano de 2010, coleta de dados de temperatura do ar e umidade relativa em 15 pontos, em dois períodos (abril e setembro), em três horários ( $8 \mathrm{~h}, 14 \mathrm{~h}$ e $20 \mathrm{~h}$ ). Os resultados mostraram que os locais com predomínio de construções com pavimento asfáltico apresentaram elevadas temperaturas e baixa umidade, revelando a importância da introdução de vegetação arbórea. Com a análise de agrupamento verificou-se que a combinação dos dados de temperatura do ar e umidade relativa forneceu um melhor agrupamento dos pontos, com uma menor distância de ligação para a formação dos grupos. Salienta-se que o uso da técnica de agrupamentos mostrou-se satisfatória para o zoneamento microclimático do campus de Cuiabá da Universidade Federal de Mato Grosso, podendo ser empregada em escala microclimática.

Palavras-chave: Microclima. Variabilidade. Agrupamento.

\begin{abstract}
:
Understanding the variability of climate elements in time and space is fundamental to the knowledge of the dynamics of microclimate. Thus, the objective was to analyze the variability of air temperature and relative humidity on the Cuiabá campus of the Federal University of Mato Grosso, and, through the clustering technique, to analyze the formation of groups to propose a zoning microclimate in the area study. To this

\footnotetext{
${ }^{1}$ Doutorando em Ciências da Engenharia Ambiental pela Universidade de São Paulo (USP). Mestre em Física Ambiental pela Universidade Federal de Mato Grosso, Cuiabá, MT, Brasil. E-mail: elisdener@usp.br

${ }^{2}$ Doutor em Agricultura Tropical pela Universidade Federal de Mato Grosso (UFMT) pós-doutorado na California State University, San Marcos, Estados Unidos da América. Professor do Instituto de Física da Universidade Federal de Mato Grosso, Cuiabá, MT, Brasil. E-mail: marcelo@pgfa.ufmt.br
} 
end, collection data of air temperature and relative humidity at 15 points in two seasons (April to September) at three times (8h, 14h and 20h) was held in 2010. The results showed that sites with a predominance of buildings with asphalt pavement had high temperatures and low humidity, revealing the importance of introducing trees. The cluster analysis showed that the combination of data of air temperature and relative humidity provides a better grouping of the points with a smaller distance connection to the formation of groups. It is noted that the use of the clustering technique was satisfactory for the microclimatic zoning at the Cuiabá campus of Universidade Federal de Mato Grosso, which can be used in microclimatic scale.

Keywords: Microclimate. Variability. Clustering.

\section{Resumen:}

Entender la variabilidad de los elementos del clima en el tiempo y el espacio es fundamental para entender la dinámica de microclimas. Así, el objetivo de este trabajo fue analizar la variabilidad de la temperatura ambiente y la humedad relativa en el campus de Cuiabá de la Universidad Federal de Mato Grosso, y por medio de la técnica de agrupamiento, analizar la formación de grupos para proponer un microclima y las diferentes zonas que lo componen dentro del área estudiada. Con este fin, en 2010 fueron registrados datos de la temperatura ambiente y de la humedad relativa en 15 puntos en dos épocas del año (abril y septiembre) en tres horarios (8hs, 14hs y 20hs). Los resultados mostraron que los lugares con un predominio de edificios con hormigón armado había altas temperaturas y baja humedad, revelando la importancia de la introducción de árboles. El análisis por agrupamiento mostró que la combinación de datos de temperatura ambiente y de humedad relativa proporciona una mejor agrupación de los puntos con una conexión de menor distancia de conexión para la formación de grupos. Cabe señalar que el uso de la técnica por agrupamiento fue satisfactoria para la distinción de zonas de microclima en el campus de Cuiabá de la Universidad Federal de Mato Grosso, que puede ser utilizada en la escala de microclima.

Palabras clave: Microclima. Variabilidad. Agrupamiento.

\section{INTRODUÇÃO}

Atualmente, não é consenso na comunidade científica que as mudanças climáticas globais sejam oriundas das atividades antropogênicas (MOLION, 2008).

Todavia, as mudanças climáticas em escala regional e local são bem documentadas, o exemplo mais significativo ocorre no ambiente urbano, no qual diversos estudos têm mostrado que as cidades criam um clima típico, decorrente dos diferentes tipos de uso e ocupação do solo (OFFERLE et al., 2005; COUTTS et al., 2007; ALVES e SPECIAN, 2009).

Nas cidades as modificações do uso do solo decorrem da concentração de construções, impermeabilização do solo com o asfaltamento de ruas e avenidas, aumento do fluxo de pessoas e automóveis, além da diminuição da vegetação natural, fazendo com que essas alterações tenham uma repercussão não só em nível local, mas em uma dimensão regional. 
Essa utilização dos solos sem planejamento, segundo Monteiro (2003), origina vários problemas, entre eles, poluição do ar, ilhas de calor, inundações no espaço urbano, refletindo as peculiaridades do clima da cidade.

Um importante instrumento para a regulação do clima urbano, principalmente no controle da poluição atmosférica, na amenização da temperatura, no aumento da umidade, são as áreas verdes urbanas que proporcionam uma melhoria da qualidade de vida das pessoas (SOUCH e GRIMMOND, 2006; ZOULIA, 2009; SHASHUA-BAR et al., 2010) .

No entanto, nos planejamentos das cidades, não se tem dado a devida atenção à questão climática. Na maioria dos centros urbanos tem se privilegiado o construir sem a preocupação de como essas construções modificam a atmosfera urbana.

No local de estudo, Universidade Federal de Mato Grosso/Cuiabá, o planejamento do campus não considerou as peculiaridades climáticas da região, a arquitetura dos institutos e faculdades foi elaborada de acordo com as construções da região sudeste, esse fato torna-se evidente na falta de ventilação cruzada.

Nesse sentido, o clima constitui-se numa das dimensões do ambiente urbano e seu estudo pode oferecer importantes contribuições ao equacionamento da questão ambiental das cidades. As condições climáticas dessas áreas, entendidas como clima urbano, são derivadas da alteração da paisagem natural e da sua substituição por um ambiente construído, palco de intensas atividades humanas (MENDONÇA, 2003).

Dessa forma, o objetivo deste trabalho foi de analisar a variabilidade da temperatura do ar e da umidade relativa em escala microclimática e, por meio da técnica de agrupamento, analisar a formação dos grupos em relação à temperatura do ar e a umidade relativa, assim como dos grupos resultantes da combinação das duas variáveis, para propor um zoneamento microclimático no campus de Cuiabá da Universidade Federal de Mato Grosso.

\section{ÁREA DE ESTUDO: A UNIVERSIDADE FEDERAL DE MATO GROSSO}

O local de estudo corresponde ao campus de Cuiabá da Universidade Federal de Mato Grosso (UFMT), com altitude média de $175 \mathrm{~m}$, localizado entre as Latitudes $-15,604^{\circ}$ e $-15,616^{\circ}$, e entre as Longitudes $-56,057^{\circ}$ e $-56,074^{\circ}$ (Figura 1 ). 
Nota-se que o campus de Cuiabá da UFMT apresenta um ambiente heterogêneo, com diferentes padrões de uso e ocupação do solo, dentre os quais se destaca as áreas verdes (bosques) que são utilizadas pelos estudantes e visitantes, para esporte e lazer. O zoológico que possui uma lagoa com vegetação. Campo de futebol que apresenta elevada área aberta com vegetação rasteira (gramado) circundada por uma pista de corrida. Áreas expostas (solo nu) sem presença de vegetação. Áreas impermeabilizadas, como estacionamentos, vias pavimentadas, faculdades e institutos.

Observa-se que as construções foram dispostas sem qualquer estudo relacionado com a climatologia urbana. Atribui-se a isso a falta de profissionais capacitados para realizar um estudo aprofundado das condições existentes no campus, assim como da falta de interesse do poder público em melhorar as condições atuais.

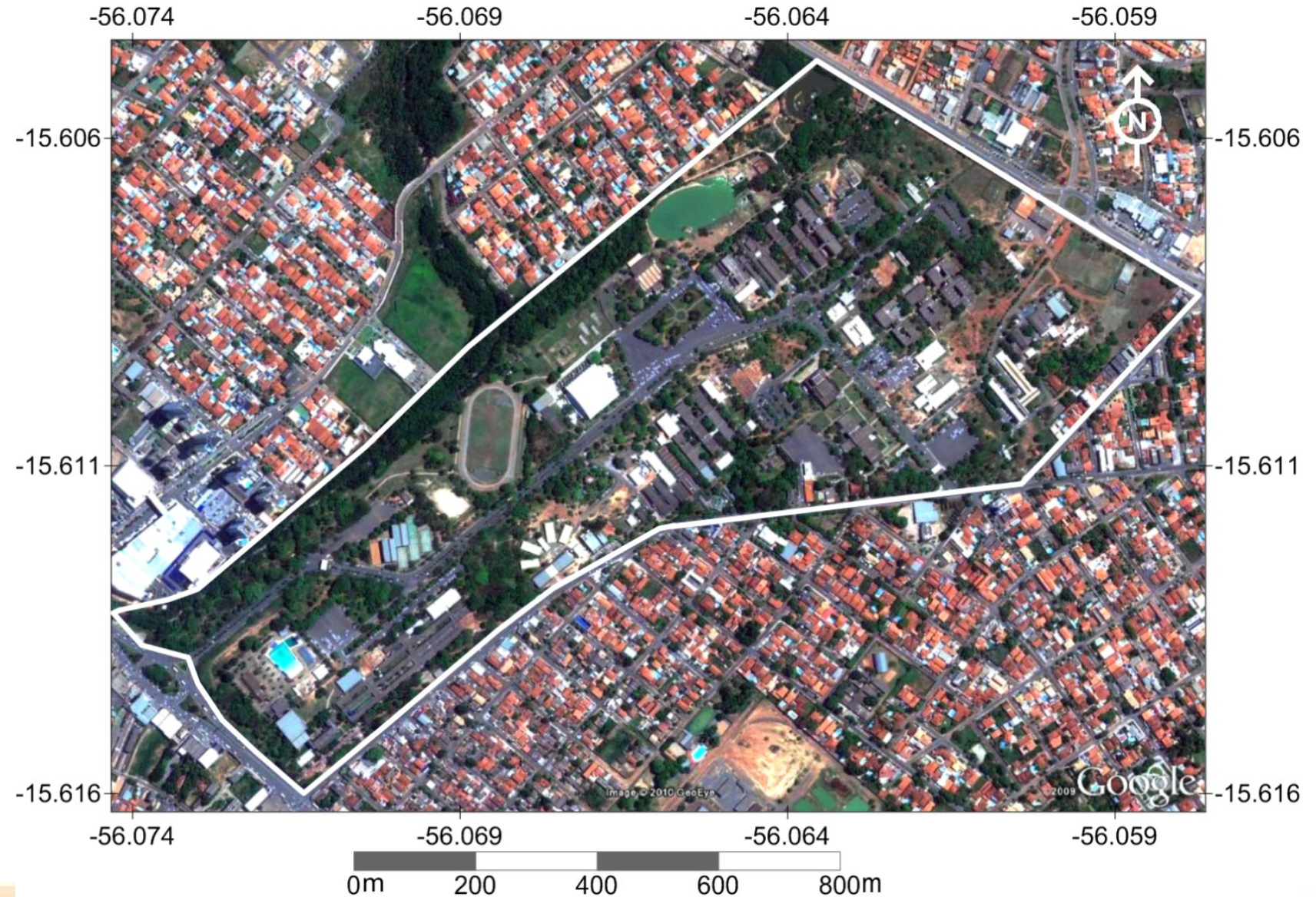

Figura 1: Mapa de localização do campus de Cuiabá da Universidade Federal de Mato Grosso. 


\section{PROCEDIMENTOS}

METODOLÓGICOS:

DA

VARIABILIDADE

AO AGRUPAMENTO

As coletas de dados (temperatura do ar e umidade relativa) foram realizadas no ano de 2010, em dois períodos: período de 19 a 29 de abril - final do período chuvoso -, e 08 a 17 de setembro - período seco -, em três horários: 8h, 14h e 20h. Foram utilizados dois termo-higrômetros digitais (HT-4000, ICEL Manaus) e um GPS (76CSx, Garmin) (Figura 2). Nas coletas tomou-se alguns cuidados, como: colocação de superfície de isopor acima do aparelho, tendo sido coletado os dados a $1.5 \mathrm{~m}$ acima do solo.

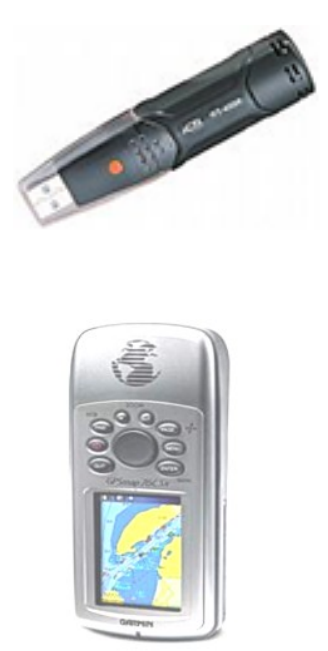

\section{Termo-Higrômetro com Data Logger}

Fabricante: ICEL Manaus;

. Modelo: HT-4000;

. Resolução: $0,1^{\circ}$ e $0,1 \%$;

. Escala: $-40^{\circ} \mathrm{C}$ a $70^{\circ} \mathrm{C} ; 0 \%$ a $100 \%$;

. Capacidade de data logger automático: 32000 leituras;

. Efetua o cálculo de IBUTG interno e externo automaticamente;

. Taxa de resposta: umidade: 5 segundos, temperatura: 20 segundos.

Fabricante: Garmin;

\section{GPS - GPSMAP}

. Modelo: 76CSx;

. Precisão do GPS: Posição: < 10 metros;

- Altimetria: Precisão de 3 metros com calibração adequada (pelo usuário e/ou pela calibração automática);

. Taxa de Atualização: 1/segundo, contínua;

- Computador de viagem: odômetro, tempo parado, tempo em andamento e velocidade média.

Figura 1: Características dos equipamentos de medições.

Escolheu-se 15 pontos para as coletas, procurou-se por pontos que se diferenciavam, principalmente, nos tipos de uso e ocupação do solo (Figura 3). 


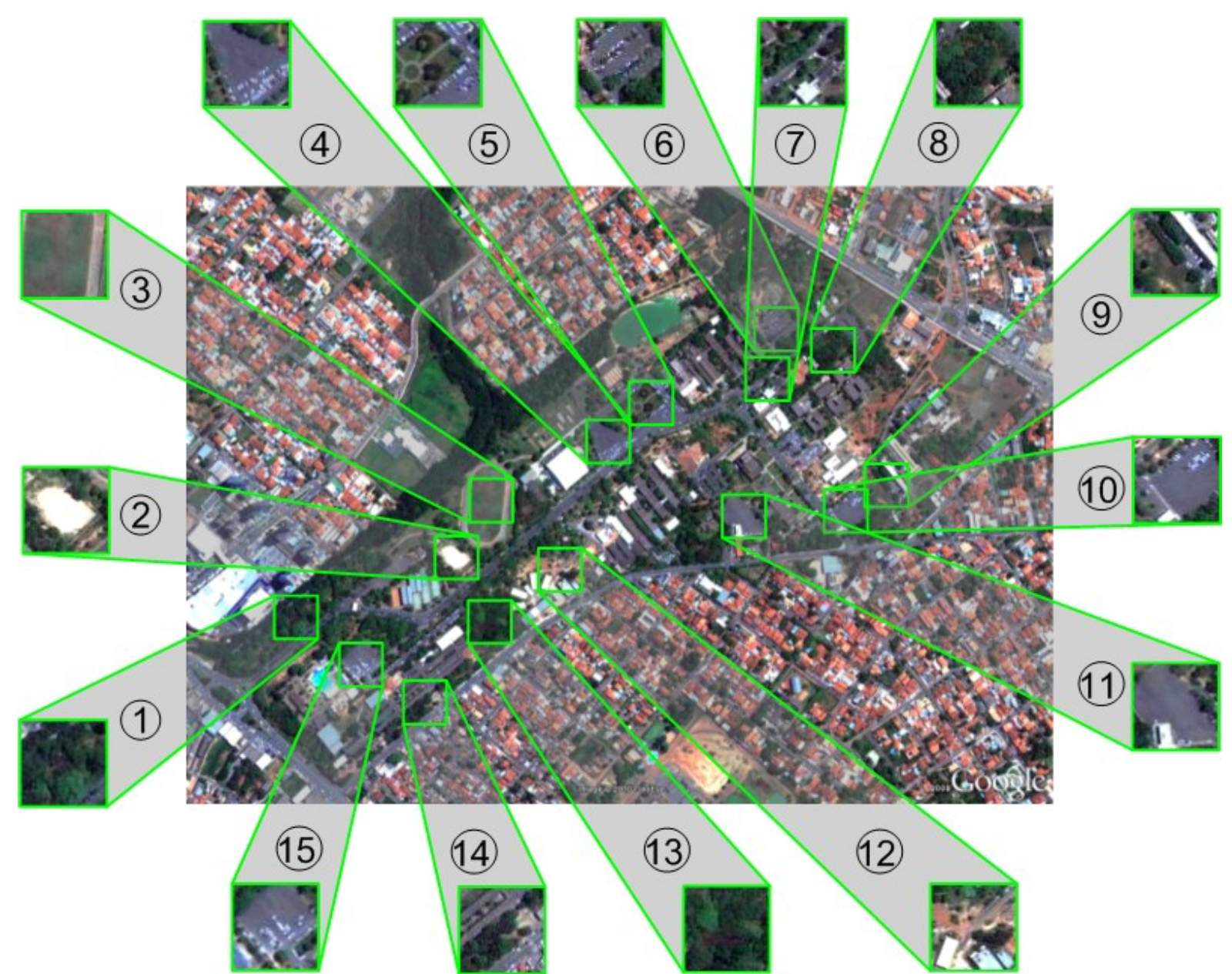

Figura 3: Representação esquemática dos pontos de coleta.

No quadro 1 verifica-se os diferentes usos do solo dos pontos de coleta, em que os pontos $4,6,10,11$ e 15 apresentam predominantemente pavimento asfáltico, os pontos 1, 8 e 13, vegetação arbórea. Enquanto que os pontos 2 (campo de areia), 3 (campo de grama), 5 (grama e pavimento asfáltico), 7 (pavimento asfáltico com árvores), 9 (área construída com grama), 12 (solo nu com construções) e 14 (área construída com árvores) não se correlacionam.

\begin{tabular}{|c|c|c|c|c|c|}
\hline \multicolumn{1}{|c|}{ Quadro 1: Características dos pontos de coleta. } \\
\hline PONTOS & \multicolumn{2}{|c|}{ COORDENADAS } & ALTITUDE & CARACTERISTICAS \\
\hline 1 & & $15^{\circ} 36^{\prime} 47^{\prime \prime}$ & $56^{\circ} 04^{\prime} 20^{\prime \prime}$ & 165 & $\begin{array}{c}\text { Vegetação predominante } \\
\text { arbórea }\end{array}$ \\
\hline 2 & $15^{\circ} 36^{\prime} 41^{\prime \prime}$ & $56^{\circ} 04^{\prime} 08^{\prime \prime}$ & 169 & $\begin{array}{c}\text { Campo de areia } \\
\text { circundado por árvores }\end{array}$ \\
\hline 3 & & $15^{\circ} 36^{\prime} 38^{\prime \prime}$ & $56^{\circ} 04^{\prime} 06^{\prime \prime}$ & 166 & $\begin{array}{c}\text { Campo de futebol } \\
\text { composto por grama }\end{array}$ \\
\hline
\end{tabular}

R. Inter. Interdisc. INTERthesis, Florianópolis, v.9, n.2, p.139-156, Jul./Dez. 2012 


\begin{tabular}{|c|c|c|c|c|}
\hline 4 & $15^{\circ} 36^{\prime} 34^{\prime \prime}$ & $56^{\circ} 03^{\prime} 58^{\prime \prime}$ & 176 & $\begin{array}{l}\text { Estacionamento } \\
\text { (pavimento asfáltico) }\end{array}$ \\
\hline 5 & $15^{\circ} 36^{\prime} 31^{\prime \prime}$ & $56^{\circ} 03^{\prime} 56^{\prime \prime}$ & 176 & $\begin{array}{l}\text { Praça composta por } \\
\text { grama e pavimento } \\
\text { asfáltico }\end{array}$ \\
\hline 6 & $15^{\circ} 36^{\prime} 28^{\prime \prime}$ & $56^{\circ} 03^{\prime} 46^{\prime \prime}$ & 174 & $\begin{array}{c}\text { Estacionamento } \\
\text { (pavimento asfáltico) } \\
\text { circundado por árvores }\end{array}$ \\
\hline 7 & $15^{\circ} 36^{\prime} 29^{\prime \prime}$ & $56^{\circ} 03^{\prime} 46^{\prime \prime}$ & 178 & $\begin{array}{c}\text { Pavimento asfáltico com } \\
\text { árvores }\end{array}$ \\
\hline 8 & $15^{\circ} 36^{\prime} 28^{\prime \prime}$ & $56^{\circ} 03^{\prime} 42^{\prime \prime}$ & 178 & $\begin{array}{c}\text { Vegetação predominante } \\
\text { arbórea }\end{array}$ \\
\hline 9 & $15^{\circ} 36^{\prime} 37^{\prime \prime}$ & $56^{\circ} 03^{\prime} 39^{\prime \prime}$ & 183 & $\begin{array}{c}\text { Área construída com } \\
\text { gramado }\end{array}$ \\
\hline 10 & $15^{\circ} 36^{\prime} 38^{\prime \prime}$ & $56^{\circ} 03^{\prime} 41^{\prime \prime}$ & 184 & $\begin{array}{l}\text { Estacionamento } \\
\text { (pavimento asfáltico) }\end{array}$ \\
\hline 11 & $15^{\circ} 36^{\prime} 38^{\prime \prime}$ & $56^{\circ} 03^{\prime} 48^{\prime \prime}$ & 178 & $\begin{array}{c}\text { Estacionamento } \\
\text { (pavimento asfáltico) }\end{array}$ \\
\hline
\end{tabular}

R. Inter. Interdisc. INTERthesis, Florianópolis, v.9, n.2, p.139-156, Jul./Dez. 2012 


\begin{tabular}{|c|c|c|c|c|c|}
\hline \multicolumn{1}{|c|}{ Quadro 1: Características dos pontos de coleta. } \\
\hline 12 & $15^{\circ} 36^{\prime} 42^{\prime \prime}$ & $56^{\circ} 04^{\prime} 02^{\prime \prime}$ & 168 & $\begin{array}{c}\text { Área de solo nu com } \\
\text { construções }\end{array}$ \\
\hline 14 & $15^{\circ} 36^{\prime} 47^{\prime \prime}$ & $56^{\circ} 04^{\prime} 05^{\prime \prime}$ & 170 & $\begin{array}{c}\text { Vegetação predominante } \\
\text { arbórea }\end{array}$ \\
\hline 15 & & $15^{\circ} 36^{\prime} 51^{\prime \prime}$ & $56^{\circ} 04^{\prime} 10^{\prime \prime}$ & 172 & $\begin{array}{c}\text { Área construída com } \\
\text { árvores }\end{array}$ \\
\hline
\end{tabular}

Com o intuito de verificar a variabilidade da temperatura do ar e da umidade relativa, após as coletas de dados, foi estruturado um banco de dados em função dos horários das medições, nos quais foram confeccionados gráficos no software Grapher 8.

A análise de agrupamento foi utilizada para se elaborar um zoneamento microclimático, partindo dos estudos de Stoeva e Stoev (2005) sobre zonas térmicas e Lobo et al (2009), zoneamento microclimático de cavernas.

A análise de agrupamentos tem como objetivo maximizar as semelhanças entre os dados, no caso entre os pontos, criando grupos distintos, bem como diferenciar esses grupos. Essa técnica vem sendo utilizada para diversas pesquisas climatológicas, sendo aplicada, principalmente, na identificação de regiões homogêneas de temperaturas (DINIZ et al., 2003; LOBO et al., 2009).

Para o agrupamento dos pontos empregou-se o método de Ward com distância euclidiana, que se caracteriza pelo procedimento de agrupamento hierárquico no qual a similaridade usada para juntar agrupamentos é calculada como a soma dos quadrados entre dois agrupamentos somados sobre todas as variáveis. Este método foi preferido pelo fato de que seu enfoque é sobre a variabilidade dos dados, de forma que os agrupamentos sejam criados com o menor acréscimo de variabilidade (LANDIM, 2003). Para tanto foi utilizado o software Statistica 8, no qual se testou vários conjuntos de dados (dados brutos, máximo, mínimo e amplitude) de temperatura do ar e da umidade relativa, separadamente, e em conjunto. Sendo que para a combinação dos dados de temperatura do ar e umidade relativa foi necessária a padronização dos dados, pois os mesmos possuem escalas diferentes. 


\section{VARIABILIDADE DA TEMPERATURA DO AR E DA UMIDADE RELATIVA}

Em relação à variabilidade da temperatura do ar, em abril e setembro (Figura 4 ), nota-se que os maiores valores de temperatura do ar ocorreram em setembro. Observa-se que os pontos que apresentaram os menores e maiores valores de temperatura do ar às $8 \mathrm{~h}$, não apresentaram o mesmo padrão às $14 \mathrm{~h}$, no entanto às $20 \mathrm{~h}$, o padrão das $8 \mathrm{~h}$ voltou a se configurar nos pontos.

Em abril (Figura 4A), nas coletas das $8 \mathrm{~h}$ e 20h, o ponto 7 (pavimento asfáltico com árvores) apresentou os maiores valores de temperatura do ar, normalmente tem-se observado nos estudos de climatologia urbana que os locais com maior presença de cobertura artificial (concreto, asfalto etc.) tendem a apresentar, no período noturno, temperaturas superiores às dos outros locais, devido às propriedades dos materiais (difusividade e condutividade térmica) que demoram certo tempo para reemitirem a energia absorvida, entretanto, neste estudo observouse que assim como do período noturno, o período da manhã também teve esta configuração, provavelmente, devido a fatores não inerentes ao tipo de cobertura do solo. Enquanto que às $14 \mathrm{~h}$, o ponto 3 (campo gramado) apresentou o maior valor. Algo semelhante ao observado por Potchter et al (2006) que verificaram que um parque coberto de grama em Tel Aviv, Israel, não foi apenas mais quente que um parque coberto de árvores, mas também era mais quente que seu entorno construído. Quanto aos menores valores, estes foram verificados no ponto 12 (solo nu com construções) em todos os horários.

Em setembro (Figura 4B), às $8 \mathrm{~h}$ o ponto 15 , composto por pavimento asfáltico, apresentou o maior valor térmico. Na coleta das $14 \mathrm{~h}$ foi o ponto 11 que apresentou o maior valor (pavimento asfáltico), já às $20 \mathrm{~h}$ o ponto 1 constituído por árvores apresentou o maior valor de temperatura do ar. Nota-se que nas coletas das 8h e $14 \mathrm{~h}$ o uso do solo foi o principal responsável pela elevação das temperaturas, contudo, às $20 \mathrm{~h}$ outros fatores devem ter provocado o deslocamento de uma massa de ar quente da área urbanizada circundante para o ponto 1. Podendo ter ocorrido um processo de advecção, como já observado por Jansson (2006) em parques de Estocolmo na Suécia e Eliasson \& Upmanis (2000) em parques de Kùbenhavn na Dinamarca e Göteborg na Suécia. Sobre os menores valores de temperatura do ar, o ponto 2 (campo de areia), 5 (grama com pavimento asfáltico) 6 (pavimento 
asfáltico com árvores), apresentaram os menores valores às $8 \mathrm{~h}, 14 \mathrm{~h}$ e $20 \mathrm{~h}$ respectivamente.

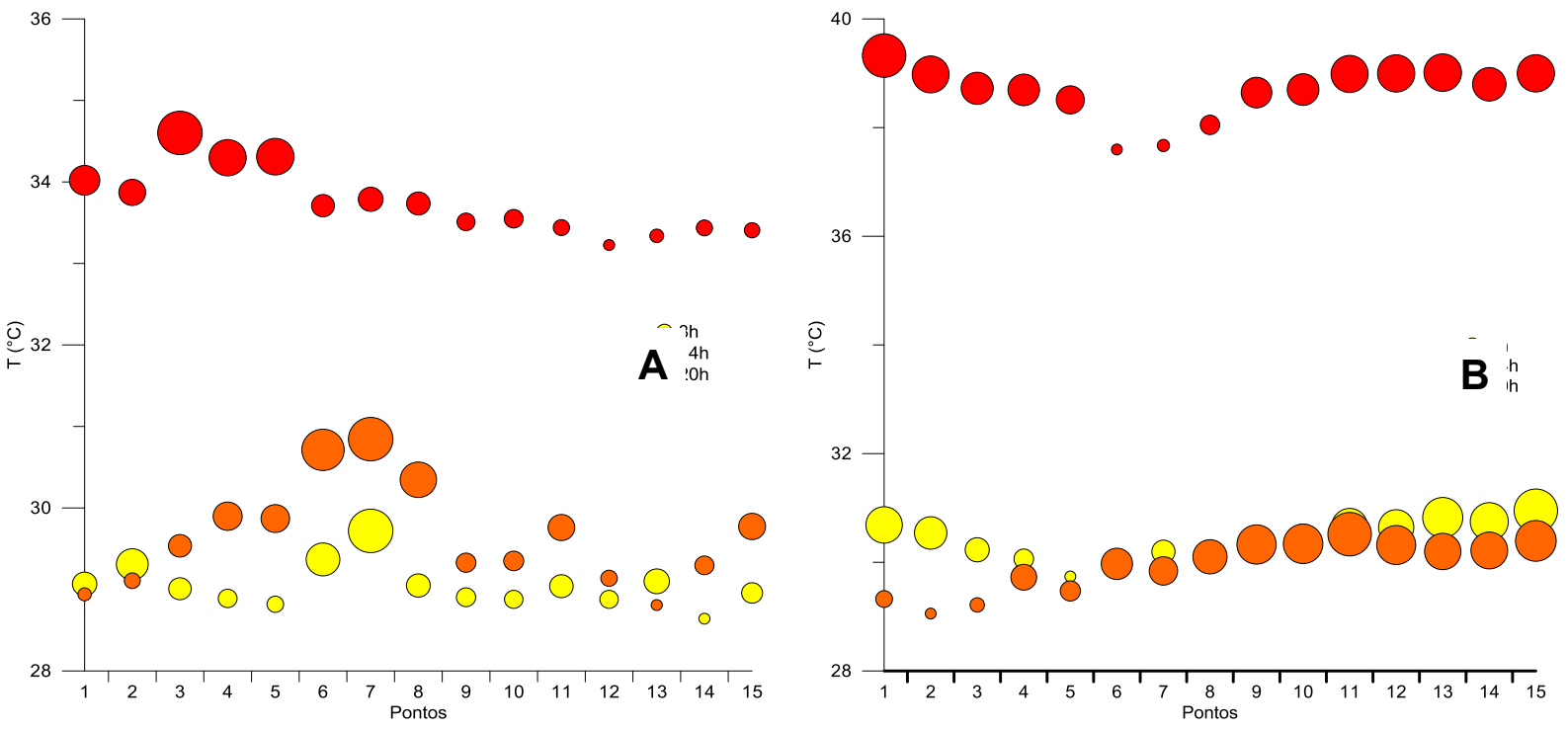

Figura 4: Variabilidade da temperatura do ar nos pontos de coleta, em abril (A) e em setembro (B).

Em relação à variabilidade da umidade relativa do ar, em abril e setembro (Figura 5), observa-se que os maiores valores de umidade relativa ocorreram em abril, devido evidentemente a maior pluviometria desse mês. Diferentemente do que fora observado para a temperatura do ar, para a umidade relativa, somente o ponto 1, em abril, apresentou maior umidade relativa em dois horários (14h e 20h).

Em abril (Figura 5A), além do ponto 1 (vegetação arbórea), os pontos 5 (grama com pavimento asfáltico) e 12 (solo nu com construções) apresentaram as maiores taxas higrométricas às $8 \mathrm{~h}$. Sobre os menores valores de umidade relativa, verifica-se que às $8 \mathrm{~h}$ e $20 \mathrm{~h}$ o ponto 7 (pavimento asfáltico com árvores) apresentou os menores valores, ao contrário do apresentado em abril para a temperatura do ar, visto que a umidade relativa é inversamente proporcional à tensão de saturação do vapor de água, que por sua vez é diretamente proporcional à temperatura do ar (TUBELIS e NASCIMENTO, 1984), às $14 \mathrm{~h}$ o ponto 14 (área construída com árvores) apresentou os menores valores higrométricos, em função, provavelmente, do seu entorno.

Em setembro (Figura 5B), assim como em abril, nas coletas das 8 h o ponto 5 (grama com pavimento asfáltico) obteve os maiores valores de umidade relativa, acredita-se que isso seja decorrente das irrigações diárias nas áreas gramadas. Às $14 \mathrm{~h}$, no ponto 7 , foi observado as menores taxas higrométricas, enquanto que às 
20h, o ponto 2, composto de areia circundado por árvores, apresentou os maiores valores. No ponto 15 (pavimento asfáltico), às $8 \mathrm{~h}$ e $14 \mathrm{~h}$, notaram-se os menores valores higrométricos, às $20 \mathrm{~h}$ foi o ponto 10 , composto também por pavimento asfáltico, que apresentou os menores valores, fato decorrido da falta de arborização nesses locais.
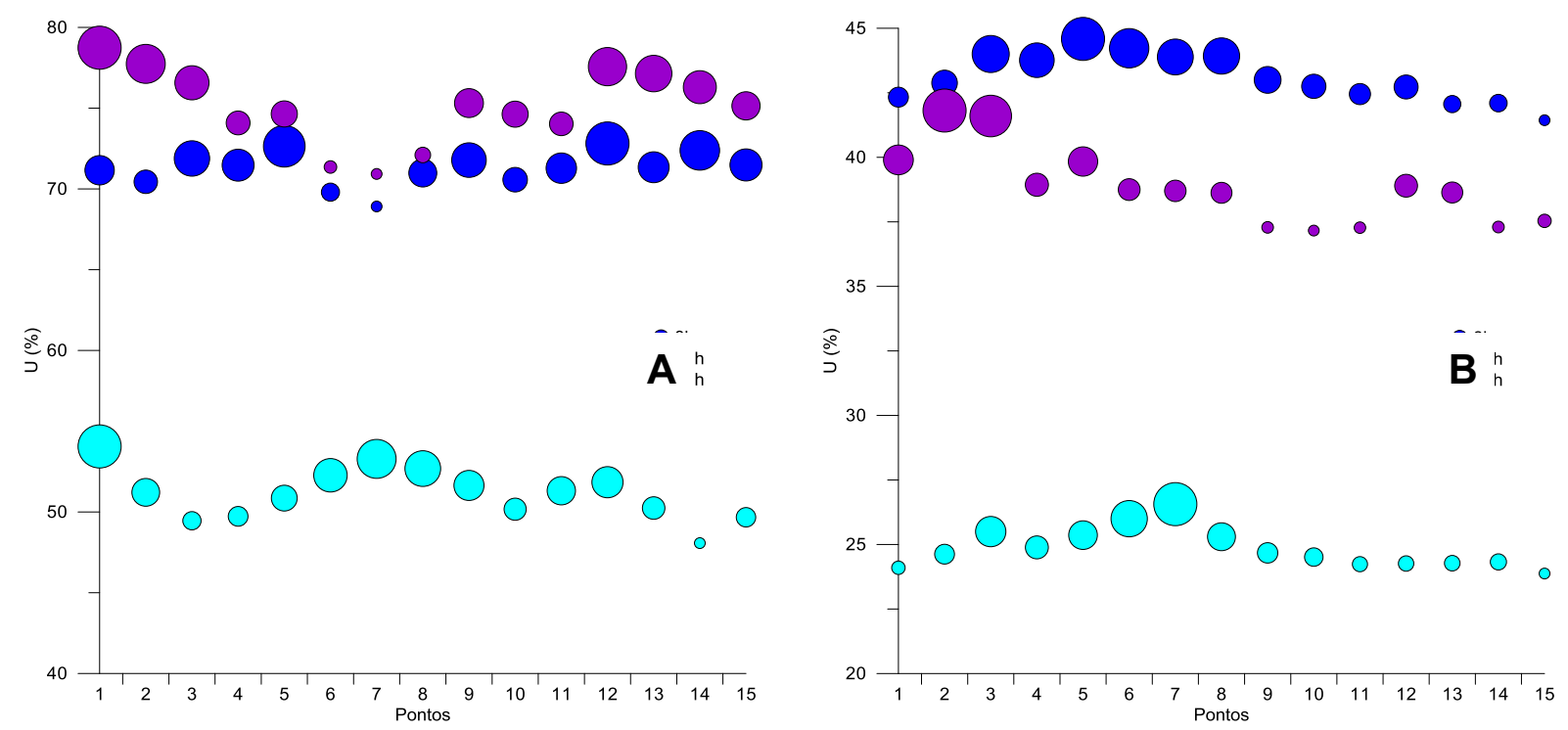

Figura 5: Variabilidade da umidade relativa do ar nos pontos de coleta, em abril (A) e em setembro (B).

\section{ANÁLISE DE AGRUPAMENTOS}

Para as análises dos agrupamentos foram confeccionados dendrogramas da temperatura do ar, da umidade relativa e da combinação das duas variáveis, com os dados brutos, amplitude diária, valor mínimo e máximo.

$\mathrm{Na}$ figura 6, observa-se a formação de diferentes grupos, na figura $6 \mathrm{~A} \circ$ dendrograma apresentou 5 grupos: grupo 1 (pontos 1 e 2); grupo 2 (pontos 3 , 4 e 5); grupo 3 (pontos 9, 10, 11, 14 e 15); grupo 4 (pontos 12 e 13) e grupo 5 (pontos 6,7 e 8). Na figura 6B nota-se a configuração de 7 grupos: grupo 1 (pontos 1 e 2); grupo 2 (pontos 4 e 5); grupo 3 (pontos 9 e 10); grupo 4 (pontos 11, 14 e 15); grupo 5 (pontos 12 e 13); grupo 6 (pontos 6 e 8 ) e grupo 7 (ponto 7 ).

No dendrograma da figura $6 \mathrm{C}$ houve a configuração de 4 grupos: grupo 1 (pontos 1, 2 e 3); grupo 2 (pontos 4, 5, 6 e 8); grupo 3 (ponto 7) e grupo 4 (pontos 9 , $10,11,12,13,14$ e 15). Na figura 6D nota-se a formação de 7 grupos: grupo 1 (ponto 1); grupo 2 (pontos 3, 4 e 5); grupo 3 (pontos 2, 11, 14 e 15); grupo 4 (pontos 
9 e 10); grupo 5 (pontos 12 e 13); grupo 6 (pontos 6 e 8) e grupo 7 (ponto 7). Com isso nota-se que o dendrograma da amplitude diária (Figura 6B) e o dendrograma do valor máximo (Figura 6D) formaram cada um 7 grupos, contudo, o dendrograma do valor máximo apresentou 2 grupos com apenas um ponto, por isso o melhor agrupamento para a temperatura do ar foi o da figura $6 \mathrm{~B}$.
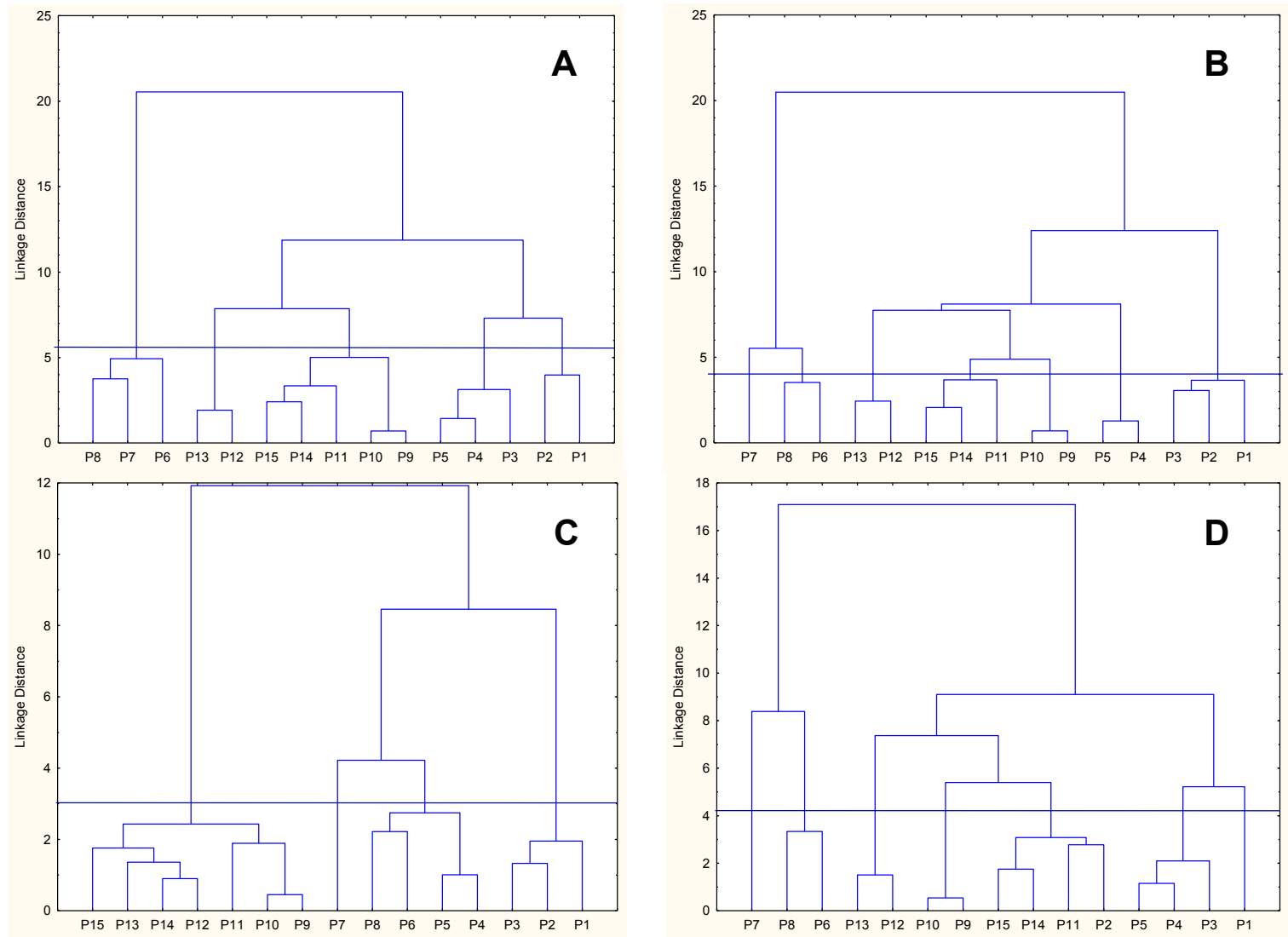

Figura 6: Dendrogramas da temperatura do ar; dados brutos (A), amplitude (B), valor mínimo (C), valor máximo (D).

Os agrupamentos da umidade relativa do ar podem ser observados na figura 7, apresentando para os dados brutos (Figura 7A) 5 grupos: grupo 1 (pontos 1 e 2); grupo 2 (pontos 4 e 5); grupo 3 (pontos 9, 10 e 11); grupo 4 (pontos 12, 13, 14 e 15) e grupo 5 (pontos 6, 7 e 8). Para a amplitude diária (Figura 7B) foi verificada a formação de 4 grupos: grupo 1 (pontos 1, 2, 3 e 4); grupo 2 (pontos 3, 12, 13, 14 e 15); grupo 3 (pontos 9, 10 e 11) e grupo 4 (pontos 6, 7 e 8).

No dendrograma da figura 7C observa-se a configuração de 4 grupos: grupo 1 (pontos 1, 2, 9,10 e 11); grupo 2 (pontos 6, 7 e 8); grupo 3 (pontos 3, 4, 5 e 14) e grupo 4 (pontos 12, 13 e 15). No dendrograma do valor máximo (Figura 7D), também verifica-se a conformação de 5 grupos: grupo 1 (pontos 1, 2, 3 e 12); grupo 
2 (pontos 4 e 5); grupo 3 (pontos 9, 10 e 11); grupo 4 (pontos 13, 14 e 15) e grupo 5 (pontos 6, 7 e 8).

O dendrograma da figura 7D apresentaram a formação de 5 grupos, sendo que o dendrograma do valor máximo formou os grupos em menor distância de ligação, portanto o mesmo configurou-se como melhor agrupamento para a umidade relativa do ar.
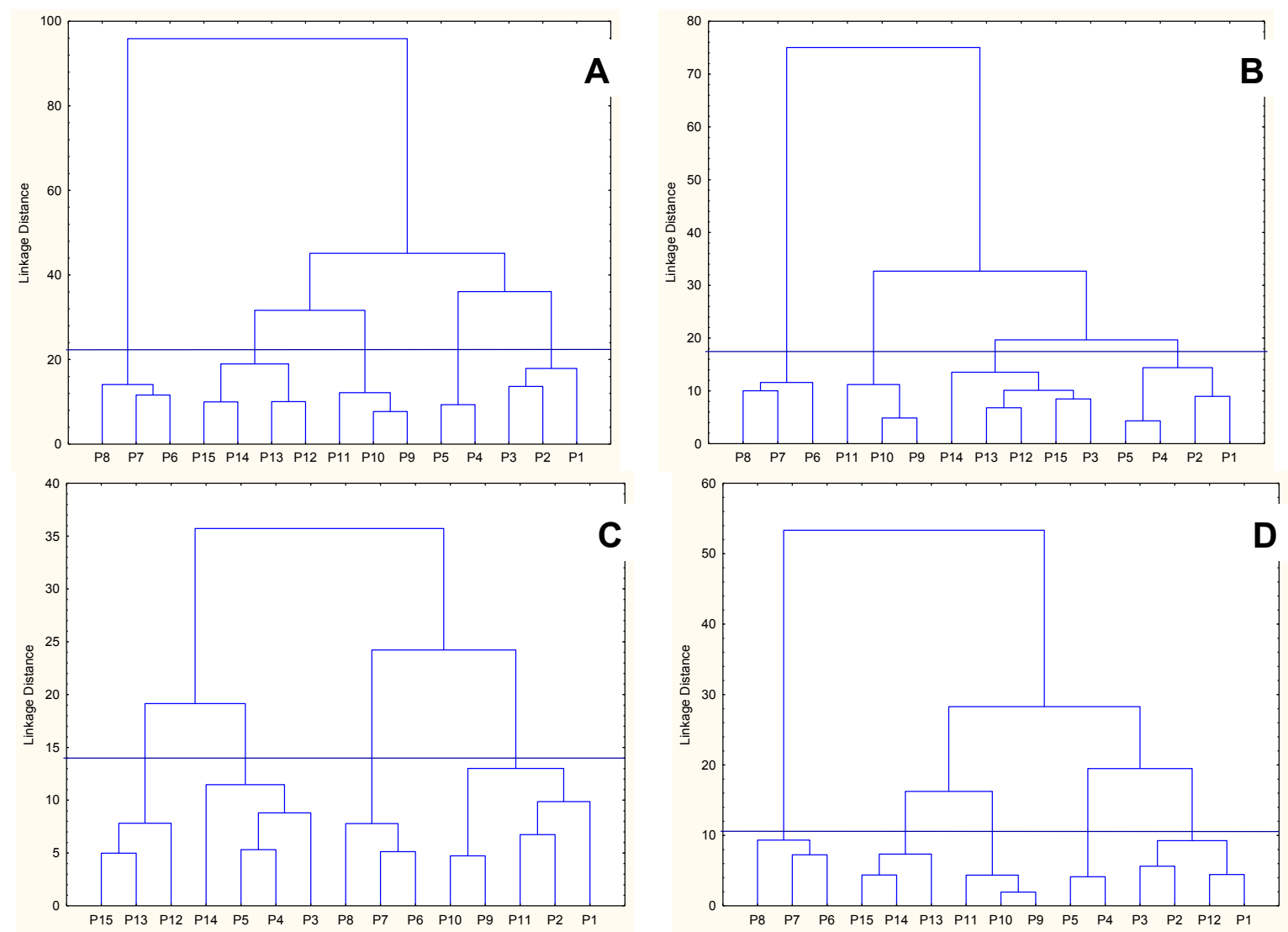

Figura 7: Dendrogramas da umidade relativa do ar; dados brutos (A), amplitude (B), valor mínimo (C), valor máximo (D).

Na figura 8 são apresentados os dendrogramas combinados da temperatura do ar com a umidade relativa. Na figura $8 \mathrm{~A}$ nota-se a configuração de 6 grupos: grupo 1 (pontos 1, 2 e 3); grupo 2 (pontos 4 e 5); grupo 3 (pontos 9, 10 e 11); grupo 4 (pontos 12, 13, 14 e 15); grupo 5 (pontos 6 e 8); e grupo 6 (ponto 7). No dendrograma da amplitude diária (Figura 8B) verifica-se a formação de 6 grupos: grupo 1 (pontos 1 e 2); grupo 2 (pontos 3 , 4 e 5); grupo 3 (pontos 12 e 13); grupo 4 (pontos 14 e 15); grupo 5 (pontos 9, 10 e 11) e grupo 6 (pontos 6, 7 e 8).

Para o valor mínimo (Figura $8 C$ ), o dendrograma apresentou 6 grupos: grupo 1 (pontos 1, 2 e 3); grupo 2 (pontos 9, 10 e 11); grupo 3 (pontos 12, 13, 14 e 15); 
grupo 4 (pontos 4 e 5); grupo 5 (pontos 6 e 8) e grupo 6 (ponto 7). Já para o valor máximo (Figura 8D), nota-se a formação de 7 grupos: grupo 1 (pontos 1 e 2); grupo 2 (pontos 11, 14 e 15); grupo 3 (pontos 12 e 13); grupo 4 (pontos 3, 4 e 5); grupo 5 (pontos 6 e 8); grupo 6 (pontos 9 e 10) e grupo 7 (ponto 7).
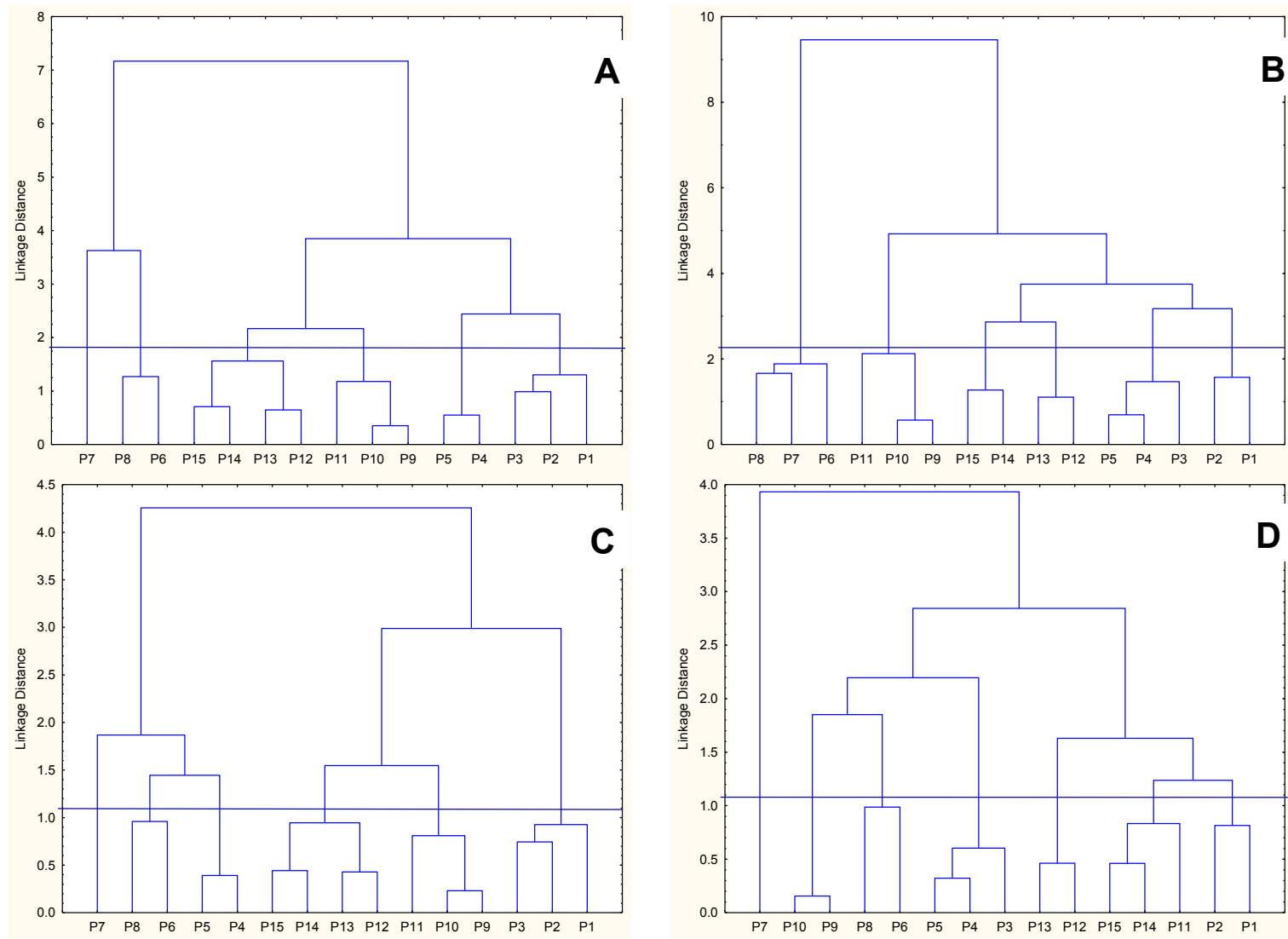

Figura 8: Dendrogramas combinados da temperatura do ar com a umidade relativa do ar; dados brutos $(A)$, amplitude (B), valor mínimo (C), valor máximo (D).

Com a combinação dos dados de temperatura do ar e umidade relativa foi possível observar melhoria gráfica nos agrupamentos, de maneira que os grupos se formaram a uma distância de ligação menor que dos agrupamentos da temperatura do ar e da umidade relativa separadamente. Verifica-se que os dendrogramas apresentaram certa similaridade, no entanto, o melhor agrupamento correspondeu ao dendrograma do valor máximo, devido ao fato de que o mesmo apresentou 7 grupos bem distribuídos com distância de ligação pequena. Portanto, o agrupamento utilizado para o zoneamento microclimático foi o dendrograma combinado do valor máximo.

Na figura 9 tem-se o zoneamento microclimático do campus de Cuiabá da Universidade Federal de Mato Grosso, elaborado a partir da análise de 
agrupamento. Verifica-se que a maior região microclimática corresponde ao grupo 2 , com $23,6 \%$ da área total, seguido do grupo $6(20 \%)$, a menor região foi formada pelo grupo 4 (3,9\%), e os grupos 1, 3, 5 e 7 obtiveram área de 13,1\%,19\%,12,2\% e $8,2 \%$, respectivamente.

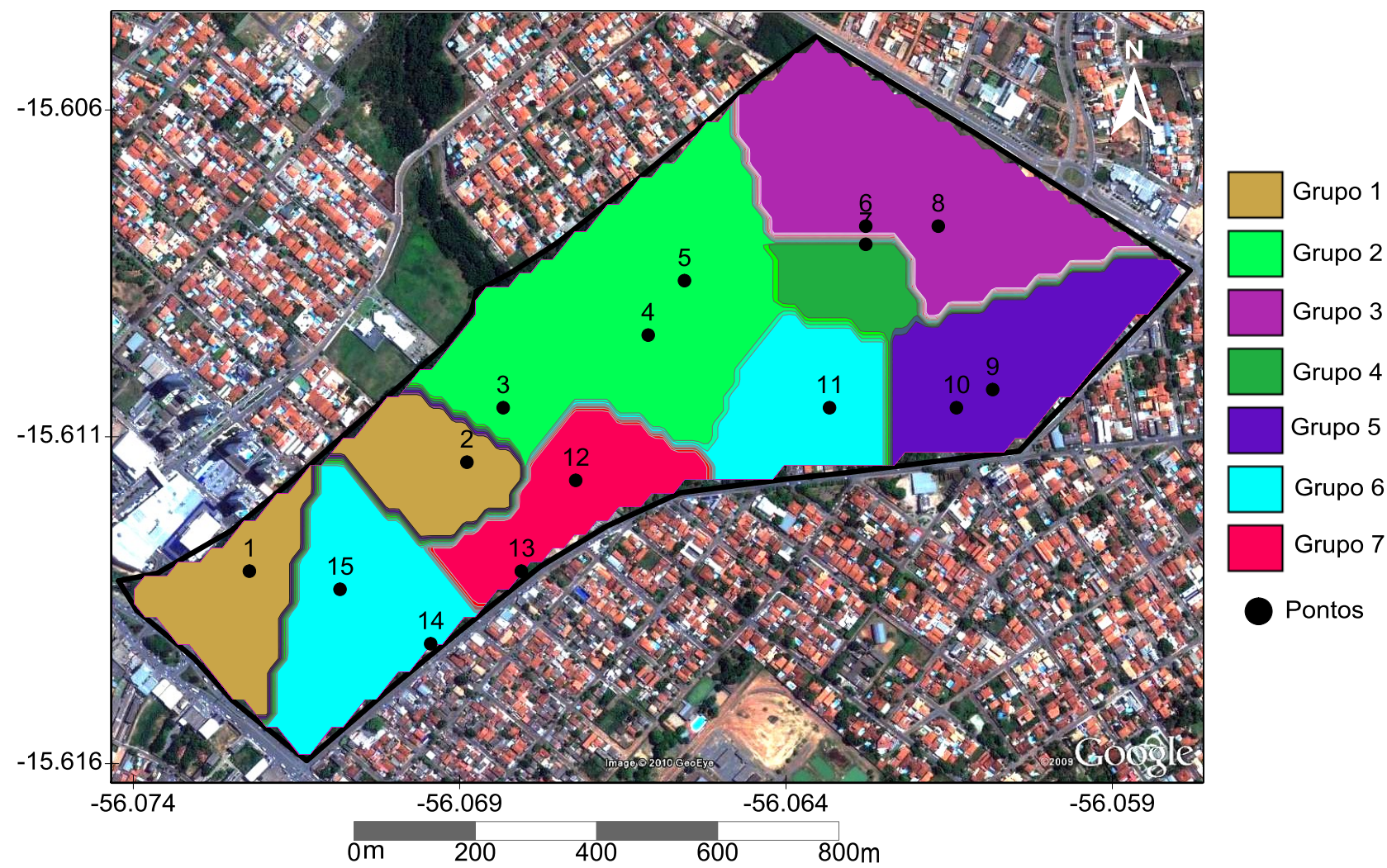

Figura 9: Zoneamento microclimático do campus de Cuiabá da Universidade Federal de Mato Grosso.

\section{CONSIDERAÇÕES FINAIS}

Em síntese, foi possível observar a variabilidade espacial (pontos de coleta) e temporal (períodos de coleta) da temperatura do ar e da umidade relativa. Notou-se que os locais com predomínio de construções com pavimento asfáltico apresentaram elevadas temperaturas e baixa umidade, revelando a importância da introdução de vegetação arbórea. A coleta de dados em períodos distintos (abril e setembro) mostrou-se importante para a análise da variabilidade temporal, pois os padrões das variáveis em estudo foram significativamente diferentes.

Com a análise de agrupamento verificou-se que a combinação dos dados de temperatura do ar e umidade relativa forneceu um melhor agrupamento dos pontos, com uma menor distância de ligação para a formação dos grupos. Salienta-se que o 
uso da técnica de agrupamentos mostrou-se satisfatória para o zoneamento microclimático do campus de Cuiabá da Universidade Federal de Mato Grosso, podendo ser empregada em escala microclimática. 


\section{REFERÊNCIAS}

ALVES, E. D. L.; SPECIAN, V. Contribuição aos estudos do clima urbano: variação térmica e higrométrica em espaços intra-urbanos. Mercator, v. 8, n. 17, p. 181-191, 2009.

COUTTS, A. M.; BERINGER, J.; TAPPER, N. J. Impact of increasing urban density on local climate: Spatial and temporal variations in the surface energy balance in Melbourne, Australia. Journal of Applied Meteorology and Climatology, v. 46, n. 4, p. 477-493, 2007.

DINIZ, G.B.; BERLATO, M.A.; CLARKE, R.T.; FONTANNA, D.C. Identificação de Regiões Homogêneas de Temperatura Máxima e Mínima do Rio Grande do Sul. Revista Brasileira de Agrometeorologia, v. 11, n. 2, p. 303-312, 2003.

ELIASSON, I.; UPMANIS, H. Nocturnal Airflow from Urban Parks-Implications for City Ventilation. Theoretical and Applied Climatology, v. 66, n. 1-2, p. 95-107, 2000.

JANSSON, C. Urban microclimate and surface hydrometeorological processes. TRITA-LWR PHD 1027. Doctoral Thesis in Land and Water Resources Sciences. KTH Architecture and the Built Environment, Stockholm. 2006, 32p.

LANDIM, P. M. B. Análise estatística de dados geológicos. 2 ed. Rio Claro: Edunesp, 2003.

LOBO, H. A. S.; PERINOTTO, J. A. de J.; POUDOU, S. Análise de agrupamentos aplicada à variabilidade térmica da atmosfera subterrânea: contribuição ao zoneamento ambiental microclimático de cavernas. Revista de estudos ambientais, v. 11, n. 1, p. 22-35, 2009.

MENDONÇA, F. Clima e planejamento urbano em Londrina: Proposição metodológica e de intervenção urbana a partir do campo termo-higrométrico. In: MONTEIRO, C. A. de F; MENDONÇA, F. Clima urbano. São Paulo: Contexto, 2003, p. 93-120.

MOLION, L. C. B. Perspectivas climáticas para os próximos 20 anos. Revista Brasileira de Climatologia, v. 3/4, n. 3, p. 117-128, 2008. 
MONTEIRO, C. A. de F. Teoria e clima urbano: um projeto e seus caminhos. In: MONTEIRO, C. A. de F; MENDONÇA, F. Clima urbano. São Paulo: Contexto, p. 967, 2003.

OFFERLE, B.; JONSSON, P.; ELIASSON, I.; GRIMMOND, C. S. B. Urban Modification of the Surface Energy Balance in the West African Sahel: Ouagadougou, Burkina Faso. Journal of Climate, v. 18, n. 19, p. 3983-3995, 2005.

POTCHTER, O.; COHEN, P.; BITAN, A. Climatic behavior of various urban parks during hot and humid summer in the Mediterranean city of Tel Aviv, Israel. International Journal of Climatology, v. 26, p. 1695-1711, 2006.

SHASHUA-BAR, L.; POTCHTER, O.; BITAN, A.; BOLTANSKY D.; YAAKOV, Y. Microclimate modelling of street tree species effects within the varied urban morphology in the Mediterranean city of Tel Aviv, Israel. International Journal of Climatology, v. 30, n. 1, p. 44-57, 2010.

SOUCH, C.; GRIMMOND, S. Applied climatology: urban climate. Progress in Physical Geography, v. 30, n. 2, p. 270-279, 2006.

STOEVA, P. STOEV, A. Cave air temperature response to climate and solar and geomagnetic activity. Memorie Della Societá Astronomica Italiana, v. 76, p. 10421047, 2005.

TUBELIS, A.; NASCIMENTO, F.J. L. Meteorologia descritiva: fundamentos e aplicações brasileiras. São Paulo, Editora Nobel, 1984. 374p.

ZOULIA, I.; SANTAMOURIS, M.; DIMOUDI, A. Monitoring the effect of urban green areas on the heat island in Athens. Environ. Monit. Assess, n. 156, p. 275-292, 2009. 